\title{
Síndrome de DRESS inducido por sulfasalazina
}

\section{Resumen}

El síndrome de DRESS (Drug Reaction with Eosinophilia and Systemic Symptoms), es una reacción severa inducida por fármacos que puede llegar a ser potencialmente mortal. Se han descrito mas de 50 fármacos asociados, incluyendo las sulfamidas, aunque es más frecuente su aparición por anticonvulsivantes aromáticos. Presentamos el caso de un varón de 34 años de raza negra que recibió tratamiento con sulfasalazina durante 21 días por una clínica de poliartritis. Requirió ingreso hospitalario por presentar fiebre, malestar general, rash cutáneo y alteraciones hematológicas y orgánicas. Tras 48 horas de terapia con corticoides se evidenció una respuesta con recuperación del estado general, parámetros analíticos y resolución de lesiones cutáneas.

El síndrome DRESS sigue teniendo una alta tasa de mortalidad por lo que una alta sospecha del mismo debe ser tenida en cuenta en aquellos pacientes que reciben estos fármacos.

\section{Sulfasalazine-induced DRESS syndrome}

DRESS syndrome is a severe drug-induced reaction that can be potentially lethal. There are more than 50 drugs that have been associated to its development, including sulfamides, even though it is more frequent by anticonvulsants. We present a case of a 34 year-old black male that received treatment with sulfasalazine during 21 days because of polyarthritis. He was interned in the hospital because of fever, malaise, skin rash and hematologic and organic affection. After 48-hour treatment with corticosteroids, there was a rapid amelioration of laboratory and clinical symptoms. 
DRESS syndrome keeps having a high mortality rate, which is why a high suspicion of it should be taken in mind, especially in those patients under treatment with these drugs.

Palabras clave: Síndrome DRESS, sulfasalazina, eosinofilia.

DRESS syndrome, sulfasalazine, eosinophilia.

\section{Introducción}

El síndrome de DRESS (Drug Reaction with Eosinophilia and Systemic Symptoms), es una reacción severa inducida por fármacos que puede llegar a ser potencialmente mortal. Se han descrito mas de 50 fármacos asociados, incluyendo las sulfamidas, aunque es más frecuente su aparición por anticonvulsivantes aromáticos [1]. No existe un término que defina a este síndrome, como mencionan Bocquet y cols. en 1996, donde plantean el término rash medicamentoso con eosinofilia y síntomas sistémicos (DRESS), sin embargo, posteriormente Descamps y cols (1997), Hashimoto y cols (1998) y Shiohara y cols (1998) demostraron la relación entre esta reacción medicamentosa y reactivación de infección por herpes virus 6, posteriormente este grupo de investigadores decidió acuñar el término de Sindrome de Hipersensibilidad Inducida por Drogas (SHDi) publicándolo en 2003 [2,3].

El acrónimo que acuñó Boquet et al[4] describe una entidad que incluye fiebre, erupción cutánea, alteraciones hematológicas y afección orgánica. La erupción cutánea suele aparecer entre 1 a 8 semanas luego del inicio del tratamiento. Se acompaña de fiebre alta y malestar general así como crecimiento de algunos ganglios, esplenomegalia o hepatomegalia. En sangre periférica se aprecia una leucocitosis, con eosinofilia y linfocitosis. La elevación de enzimas hepáticas de citolisis es frecuente.
Puede desarrollarse una afectación orgánica severa que incluye pancreatitis, pericarditis, hepatitis fulminante, nefropatía intersticial, entre otras llevando a su vez a fallo multiorgánico que amenaza la vida del paciente.

\section{Presentación del caso clínico}

Paciente varón de 34 años de raza negra, natural de Mauritania, sin antecedentes de interés. Acudió remitido desde urgencias por clínica de 6 días de evolución de poliartritis. Refería episodios autolimitados de artritis de tobillos de 3 días de evolución. A la exploración se evidenció artritis de carpos, rodillas y tobillo izquierdo. Con la artrocentesis de rodilla se obtuvieron $10 c c$ de líquido sinovial de aspecto inflamatorio. Se inició tratamiento con sulfasalazina (SSZ) a dosis bajas aumentando hasta 500 mg cada 12 horas y se solicitaron pruebas complementarias para completar el estudio: analítica completa con estudio inmunológico, radiografía de tórax, serologías y quantiferon para descartar la presencia de infección. Al cabo de tres semanas el paciente acudió de forma no programada por vómitos, malestar general, disgeusia e hiporexia y prurito intenso, que el paciente relacionaba con el inicio del tratamiento. Además presentaba sensación distérmica sin fiebre termometrada. La analítica mostró una alteración del perfil hepático (bilirrubina total:1.39 mg/dl (0.201.20), bilirrubina indirecta: $0.66 \mathrm{mg} / \mathrm{dl}$ (0.00-0.75), 
bilirrubina directa:0.73 mg/dl (0.00-0.50), GOT:193 ui/l (5-34), GPT:291ui/l (1-55), GGT:80ui/l (9-64), LDH:744 ui/l (125-243), con elevación de reactantes de fase aguda [PCR:67.2mg/l (0.0-10.0)] y alteración del hemograma (leucocitos:9.4 x10e9/L (4.011.3), neutrófilos:46.7\%, linfocitos:26.2\%, eosinófilos: $20.3 \%)$. El FR, los ANA y las serologías víricas y quantiferon resultaron negativos. Se le indicó al paciente que suspendiera definitivamente la SSZ, se inició tratamiento antihistamínico y se añadió tratamiento analgésico y antiinflamatorio.

A los 15 días volvió a consultar por edema localizado en tronco, cara, empeoramiento del prurito, sin presentar fiebre. A la exploración destacaban adenopatías cervicales, inguinales y axilares no dolorosas y una erupción papulosa generalizada de predominio en tronco y cara, que respetaba las palmas de las manos y las plantas de los pies.

En la analítica persistía la alteración de las enzimas hepáticas (GPT:146ui/L, CK total:323ui/l, amilasa: 28 ui/l) y en el hemograma la leucocitosis de 61.500 con eosinofilia (eosinófilos absolutos: 31.600 , eosinófilos: $51.3 \%$ ). En el frotis de sangre periférica se observó leucocitosis con eosinofilia.

Con la sospecha de síndrome de DRESS se decidió ingresar al paciente para confirmar el diagnóstico. Se inició tratamiento con glucocorticoides sistémicos $(1 \mathrm{mg} / \mathrm{kg}$ ). A las 48 horas el paciente mejoró tras presentar disminución significativa de las lesiones cutáneas, permaneciendo afebril y con mejoría de los parámetros analíticos.

\section{Discusión}

Se ha estimado que el síndrome DRESS tiene una mortalidad de alrededor del 10\%[5], por lo que los clínicos debemos tener presente esta entidad en caso de introducir un nuevo fármaco.

Su fisiopatología y etiología aún no están esclarecidas, se cree que puede existir un fenómeno inmu- nológico desencadenante en su origen, que supone liberación de citocinas por medio de linfocitos $T$ y activación de macrófagos. [6] La identificación precoz del síndrome nos permite iniciar el tratamiento adecuado minimizando las complicaciones. Actualmente, el tratamiento del síndrome de DRESS no está definido. Debe retirarse el fármaco responsable cuanto antes y se debe iniciar tratamiento con glucocorticoides [7]. Nuestro paciente presentó una buena evolución con dosis medias de prednisona oral. La recuperación puede lograrse a las pocas semanas o incluso meses después de retirar el fármaco y en pocos casos se ha visto recidiva si se vuelve a estar en contacto con el mismo fármaco [8].

\section{Responsabilidades éticas}

\section{Protección de personas y animales}

Los autores declaran que para esta investigación no se han realizado experimentos en seres humanos ni en animales.

\section{Confidencialidad de datos}

Los autores declaran haber seguido los protocolos de su centro de trabajo sobre la publicación de datos del paciente y que todos los pacientes incluidos en el estudio han recibió información suficiente y han dado su consentimiento informado por escrito para participar en dicho estudio.

\section{Derecho a la privacidad y consentimiento informado}

Los autores han obtenido el consentimiento informado del paciente referido en el artículo. Este documento obra en poder del autor de correspondencia.

\section{Conflictos de interés}

Los autores declaran no tener ningún conflicto de intereses. 


\section{Bibliografía}

1. Michel F, Navellou JC, Ferraud D, et al. DRESS syndrome in a patient on sulfasalazine for rheumatoid arthritis. Joint Bone Spine 2005;72:82-5.

2. Shiohara T, Ilijima M, Ikezawa Z, Hashimoto K. The diagnosis of a DRESS Syndrome has been sufficiently established on the basis of typical features and viral reactivations. Br J Dermatol 2007; 156(5):1083-4.

3. Kano $Y$, Shiohara T. The variable pictures of drug-induced hypersensitivity syndrome/ drug rash with eosinophilia and systemic symptoms in relation to the eliciting drug. Immunol Allergy Clin North Am 2009; 29(3): 481-501.

4. Bocquet $H$, Bagot $M$, Roujeau JC. Drug-induced pseudolymphoma and drug hypersensitivity syndrome (drug rash with eosinophilia and systemic symptoms:DRESS). Semin Cutan Med Surg 1996;1:250-7.

5. Cacoub P, Musette P, Descamps V, Meyer O, Speirs C, Finzi L, Roujeau JC. The DRESS syndrome: a literature review. Am J Med 2011;124:588-97.

6. Schnetzke V, Bossert T, Scholls S, et al. Drug-induced lymphadenopathy with eosinophilia and renal failure mimicking lymphoma disease:dramatic onset of DRESS Syndrome associated with antibiotic treatment. Ann Hematol 2011;90: 1353-5.

7. Guy Vanderstraeten, Levent Özçakar, Koenraad Verstraete; DRESS syndrome induced by sulphasalazine in rheumatoid arthritis; Letters to the editor / Joint Bone Spine 73 (2006) 763768.

8. Shear NH, Spielberg SP. Anticonvulsivants Hypersensitivity Syndrome in vitro Asessment of Risk. Am Soc Clin Invest Inc 1988;82:1826-1832.
Opina sobre este artículo:
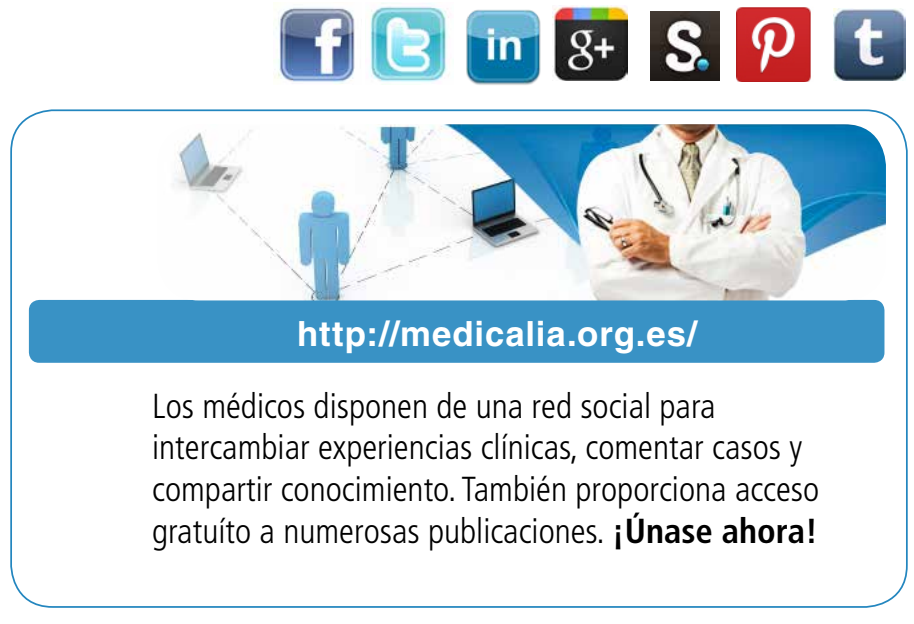

\section{Publish with iMedPub}

http://www.imed.pub

Acta Reumatológica es una revista que tiene por fin la difusión de estudios clínicos relacionados con aspectos prácticos del diagnóstico, tratamiento y seguimiento de pacientes con patología reumatológica, de estudios epidemiológicos relacionados con patología inflamatoria y musculoesquelética de presentación común o infrecuente en la práctica clínica tanto en población adulta como pediátrica, de casos clínicos de patología poco habitual o de presentaciones inhabituales de patología frecuente, de imágenes didácticas e ilustrativas en reumatología y del estado actual e innovación en la formación especializada en reumatología. 